\title{
Measurements of Consensus in Multi-granular Linguistic Group Decision-making *
}

\author{
E. Herrera-Viedma ${ }^{1}$, F. Mata ${ }^{2}$, L. Martínez ${ }^{2}$ and F. Chiclana ${ }^{3}$ \\ 1 Dept. of Computer Science and A.I., University of Granada, 18071 - Granada, \\ Spain, viedma@decsai.ugr.es \\ 2 Dept. of Computer Science, University of Jaén, 23071 - Jaén, Spain, \\ martin@ujaen.es, fmata@ujaen.es \\ ${ }^{3}$ Centre for Computational Intelligence, School of Computing, De Montfort \\ University - Leicester LE1 9BH, UK, chiclana@dmu.ac.uk
}

\begin{abstract}
The reaching of consensus in group decision-making (GDM) problems is a common task in group decision processes. In this article, we consider GDM with linguistic information. Different experts may have different levels of knowledge about a problem and, therefore, different linguistic term sets (multi-granular linguistic information) can be used to express their opinions.

The aim of this paper is to present different ways of measuring consensus in order to assess the level of agreement between the experts in multi-granular GDM problems. To make the measurement of consensus in multi-granular GDM problems possible and easier, it is necessary to unify the different linguistic term sets into a single one. This is done using fuzzy sets defined on a basic linguistic term set (BLTS). Once the information is uniformed, two types of measurement of consensus are carried out: consensus degrees and proximity measures. The first ones assess the agreement among all the experts' opinions, while the second ones are used to find out how far the individual opinions are from the group opinion. The proximity measures can be used by a moderator in the consensus process to suggest to the experts the necessary changes to their opinions in order to be able to obtain the highest degree of consensus possible. Both types of measurements are computed in the three different levels of representation of information: pair of alternatives, alternatives and experts.
\end{abstract}

Keywords: Consensus, multi-granular linguistic information, group decisionmaking, linguistic modelling, fuzzy preference relation.

\section{Introduction}

A group decision-making (GDM) problem may be defined as a decision situation where: i) there exist two or more experts that are characterized

\footnotetext{
* This work has been partially supported by the Research Project TIC2002-03348.
} 
by their own perceptions, attitudes, motivations and knowledge, ii) there exists a problem to be solved, and iii) they try to achieve a common solution.

Fuzzy sets theory has proven successful for handling fuzziness and modelling qualitative information $[6,7,13]$. In this theory, the qualitative aspects of the problem are represented by means of "linguistic variables" [14], i.e., variables whose values are not numbers but words or sentences in a natural or an appropriate artificial language.

An important parameter to determine in a linguistic context is the "granularity of uncertainty", i.e., the cardinality of the linguistic term set that will be used to express the information. Because experts may come from different research areas, and thus have different levels of knowledge, it is natural to assume that linguistic term sets of different cardinality and/or semantics could be used to express their opinions on the set of alternatives. In these cases, we say that we are working in a multi-granular linguistic context $[4,12]$, and we will call this type of problem a multigranular linguistic GDM problem.

In GDM problems there are two processes to carry out before obtaining a final solution $[3,5,8,9]$ : the consensus process and the selection process (see Figure 1). The first one refers to how to obtain the maximum degree of consensus or agreement between the set of experts on the solution set of alternatives. Normally this process is guided by the figure of a moderator $[5,9]$. The second one consists in how to obtain the solution set of alternatives from the opinions on the alternatives given by the experts. Clearly, it is preferable that the set of experts reach a high degree of consensus before applying the selection process. In [4], the selection process for multi-granular linguistic GDM problem was studied. Therefore, in this paper, we focus on the consensus process, and in particular we address the problem of how to measure the consensus in such a type of GDM problem.

Traditionally, the consensus process is defined as a dynamic and iterative group discussion process, coordinated by a moderator, who helps the experts to bring their opinions closer. In each step of this process, the moderator, by means of a consensus measure, knows the actual level of consensus between the experts which establishes the distance to the ideal state of consensus. If the consensus level is not acceptable, i.e., if it is lower than a specified threshold, then the moderator would urge the experts to discuss their opinions further in an effort to bring them closer $[2,15]$. 


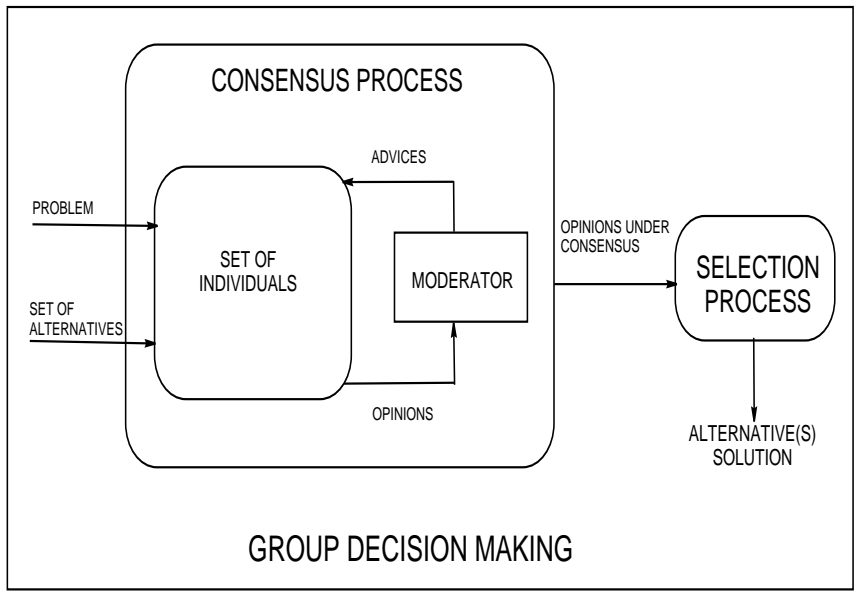

Fig. 1. Resolution process of a group decision-making problem

The aim of this paper is to present two different measurements to assess the level of agreement between the experts in multi-granular GDM problems. These measurements can be classified into two types:

a) Consensus measurement to identify the level of agreement among all experts and to decide when the consensus process should stop.

b) Proximity measurement to evaluate the distance between the experts' individual opinions and the group or collective opinion. The proximity values are used by the moderator to guide the direction of the changes in the experts' opinions in order to increase the degree of consensus.

For each one of these measurements, it is interesting not only to know the global agreement or proximity amongst experts' but also the partial degrees on a particular alternative or pair of alternatives. To do this, both types of measurements are carried out at three different levels of representation of information:

Level 1 or pair of alternatives level. At this level both the agreement amongst all the experts and the distance between each experts' individual opinion and the group opinion on each pair of alternatives are calculated.

Level 2 or alternatives level. At this level, the consensus degree and the proximity on each alternative are obtained.

Level 3 or experts' level. In this last level, the global consensus degree amongst all the experts and the distance between each individual 
expert's opinion and the group opinion on all the alternatives are calculated.

This means that in total six measurements are obtained, a consensus measure and a proximity measure at each one of the three levels. To make the computation of these six measurements in multi-granular GDM problems possible and easier, it is necessary to unify the different linguistic term sets into a single one. To do this, fuzzy sets on a basic linguistic term set (BLTS) are used, and the appropriate transformation functions are defined.

The rest of the paper is set out as follows. The multi-granular linguistic GDM problem is described in Section 2. The different consensus and proximity measures are presented in Section 3. Finally, in Section 4 we draw our conclusions.

\section{Multi-granular Linguistic GDM Problems}

We focus on GDM problems in which two or more experts express their preferences about a set of alternatives by means of linguistic labels. A classical way to express preferences in GDM problems is by means of preference relations [3]. A GDM problem based on linguistic preference relations may be defined as follows: there are $X=\left\{x_{1}, x_{2}, \ldots, x_{n}\right\}(n \geq 2)$, a finite set of alternatives, and a group of experts, $E=\left\{e_{1}, e_{2}, \ldots, e_{m}\right\}(m \geq 2)$; each expert $e_{i}$ provides his/her preferences on $X$ by means of a linguistic preference relation, $\mu_{P_{e_{i}}}: X \times X \rightarrow S$, where $S=\left\{s_{0}, s_{1}, \ldots, s_{g}\right\}$ is a linguistic term set characterized by its cardinality or granularity, $\#(S)=g+1$. Additionally, the following properties are assumed:

1. The set $S$ is ordered: $s_{i} \geq s_{j}$, if $i \geq \mathrm{j}$.

2. There is the negation operator: $\operatorname{Neg}\left(s_{i}\right)=s_{j}$ such that $j=g-i$.

3. There is the min operator: $\operatorname{Min}\left(s_{i}, s_{j}\right)=s_{i}$ if $s_{i} \leq s_{j}$.

4. There is the max operator: $\operatorname{Max}\left(s_{i}, s_{j}\right)=s_{i}$ if $s_{i} \geq s_{j}$.

The semantics of the terms is represented by fuzzy numbers defined on the $[0,1]$ interval. One way to characterize a fuzzy number is by using a representation based on parameters of its membership function [1]. For example, the following semantics, represented in Figure 2, can be assigned to a set of seven terms via triangular fuzzy numbers:

$$
\begin{aligned}
& P=\text { Perfect }=(0.83,1,1) \quad V H=\text { Very_High }=(0.67,0.83,1) \\
& H=H i g h=(0.5,0.67,0.83) M=\text { Medium }=(0.33,0.5,0.67) \\
& L=L o w=(0.17,0.33,0.5) \quad V L=\text { Very_Low }=(0,0.17,0.33) \\
& N=\text { None }=(0,0,0.17)
\end{aligned}
$$




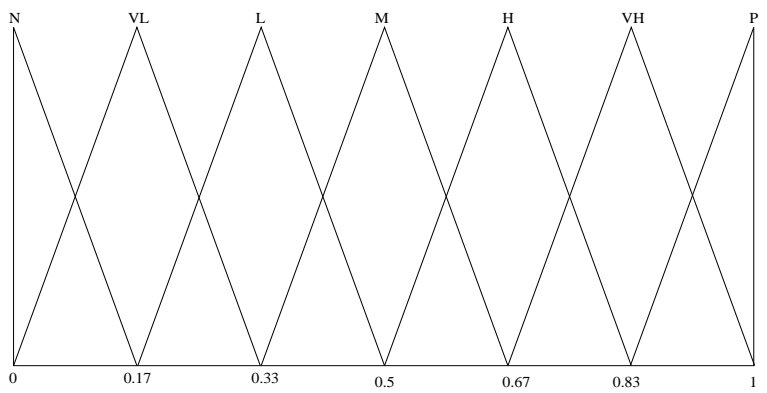

Fig. 2. A set of seven terms with their semantics

The ideal situation in GDM problems in a linguistic context would be one where all the experts use the same linguistic term set $S$ to provide their opinions. However, in some cases, experts may belong to distinct research areas and will, therefore, have different levels of knowledge about the alternatives. A consequence of this is that the expression of preferences will be based on linguistic term sets with different granularity, which means that adequate tools to manage and model multi-granular linguistic information become essential $[4,7,12]$.

In this paper, we deal with multi-granular linguistic GDM problems, i.e., GDM problems where each expert $e_{i}$ may express his/her opinions on the set of alternatives using different linguistic term sets with different cardinality $S_{i}=\left\{s_{0}^{i}, \ldots, s_{p}^{i}\right\}$, by means of a linguistic preference rela-

tion $P_{e_{i}}$, where $p_{i}^{j k} \in S_{i}$ represents the preference of alternative $x_{j}$ over alternative $x_{k}$ for that expert.

\section{The Measurement of Consensus in Multi-granular Linguistic Context}

The measurement of consensus in GDM problems is carried out using two different measures: consensus measures and proximity measures. However, as we assume multi-granular linguistic context, the first step must be to obtain a uniform representation of the preferences, i.e., experts' preferences must be transformed (using a transformation function) into a single domain or linguistic term set that we call basic linguistic term set (BLTS) and is denoted by $S_{T}$.

The measurement of consensus in multi-granular linguistic GDM problems is therefore carried out in three steps: (i) making the linguistic information uniform, (ii) computation of consensus degrees and (iii) computation of proximity measures 


\subsection{Making the Linguistic Information Uniform}

In this step, a basic linguistic term set (BLTS), $S_{T}$, has to be selected. To do this it seems reasonable to impose a granularity high enough to maintain the uncertainty degrees associated to each one of the possible domains to be unified. This means that the granularity of the BLTS has to be as high as possible. Therefore, in a general multi-granular linguistic context, to select $S_{T}$ we proceed as follows:

1. If there is only one linguistic term set, from the set of different domains to be unified, with maximum granularity, then we choose that one as the BLTS, $S_{T}$.

2. If there are two or more linguistic term sets with maximum granularity, then the election of $S_{T}$ will depend on the semantics associated to them:

(a) If all of them have the same semantics (with different labels), then any one of them can be selected as $S_{T}$.

(b) If two or more of them have different semantics, then $S_{T}$ is defined as a generic linguistic term set with a number of terms greater than the number of terms a person is able to discriminate, which is normally 11 or 13 [11], although we can find cases of BLTS with 15 terms symmetrically distributed $[4,10]$.

Once $S_{T}$ has been selected, the following multi-granular transformation function is applied to transform every linguistic value into a fuzzy set defined on $S_{T}$ :

Definition 1 [4] If $A=\left\{l_{0}, \ldots, l_{p}\right\}$ and $S_{T}=\left\{c_{0}, \ldots, c_{g}\right\}$ are two linguistic term sets, with $g \geq p$, then a multi-granular transformation function, $\tau_{A S_{T}}$, is defined as

$$
\begin{gathered}
\tau_{A S_{T}}: A \longrightarrow F\left(S_{T}\right) \\
\tau_{A S_{T}}\left(l_{i}\right)=\left\{\left(c_{h}, \alpha_{i h}\right) / h \in\{0, \ldots, g\}, \forall l_{i} \in A\right. \\
\alpha_{i h}=\max _{y} \min \left\{\mu_{l_{i}}(y), \mu_{c_{h}}(y)\right\}
\end{gathered}
$$

where $F\left(S_{T}\right)$ is the set of fuzzy sets defined on $S_{T}$, and $\mu_{l_{i}}(y)$ and $\mu_{c_{h}}(y)$ are the membership functions of the fuzzy sets associated to the linguistic terms $l_{i}$ and $c_{h}$, respectively.

The composition of the linguistic preference relations provided by the experts $\mu_{P_{e_{i}}}$ with the multi-granular transformation functions $\left\{\tau_{S_{i} S_{T}}, \forall i\right\}$ 
will result in a unification of the preferences for the whole group of experts. In particular, the linguistic preference $p_{i}^{l k}$ will be transformed into the fuzzy set, defined on $S_{T}=\left\{c_{0}, \ldots, c_{g}\right\}$,

$$
\begin{gathered}
\tau_{S_{i} S_{T}}\left(p_{i}^{l k}\right)=\left\{\left(c_{h}, \alpha_{i h}^{l k}\right) / h=0, \ldots, g\right\} \\
\alpha_{i h}^{l k}=\max _{y} \min \left\{\mu_{p_{i}^{l k}}(y), \mu_{c_{h}}(y)\right\} .
\end{gathered}
$$

We will continue to denote $\tau_{S_{i} S_{T}}\left(p_{i}^{l k}\right)$ by $p_{i}^{l k}$, and we will use only the membership degrees to denote the uniformed linguistic preference relation:

$$
\mathbf{P}_{\mathbf{e}_{\mathbf{i}}}=\left(\begin{array}{ccc}
p_{i}^{11}=\left(\alpha_{i 0}^{11}, \ldots, \alpha_{i g}^{11}\right) & \cdots & p_{i}^{1 n}=\left(\alpha_{i 0}^{1 n}, \ldots, \alpha_{i g}^{1 n}\right) \\
\vdots & \ddots & \vdots \\
p_{i}^{n 1}=\left(\alpha_{i 0}^{n 1}, \ldots, \alpha_{i g}^{n 1}\right) & \cdots & p_{i}^{n n}=\left(\alpha_{i 0}^{n n}, \ldots, \alpha_{i g}^{n n}\right)
\end{array}\right)
$$

\subsection{Computation of Consensus Degrees}

In GDM problems, each consensus parameter requires the use of a dissimilarity function to obtain the level of agreement among all the experts. Several dissimilarity functions have been proposed to measure how far each individual expert is from the rest, including the Euclidean distance, the cosine and sine of the angle between vectors, etc $[2,15]$.

Initially, we used these traditional distance functions to measure the proximity between the linguistic preferences $p_{i}^{l k}, p_{j}^{l k}$ given by experts $e_{i}, e_{j}$, by comparing the membership degrees vectors associated to them. However, after checking the results of some trials, we discovered cases in which unexpected results were obtained, as is shown in the following example, which implied that these functions were not suitable for our objectives.

Example 1 If $p_{1}^{12}=(1,0,0,0,0,0), p_{2}^{12}=(0,0,0,1,0,0)$ and $p_{3}^{12}=$ $(0,0,0,0,0,1)$ are three experts' assessments on the pair of alternatives $\left(x_{1}, x_{2}\right)$, the following values are obtained using the Euclidean distance:

$$
d\left(p_{1}^{12}, p_{2}^{12}\right)=\sqrt{\sum_{i=0}^{g}\left(\alpha_{1 i}^{12}-\alpha_{2 i}^{12}\right)^{2}}=\sqrt{2} \quad ; \quad d\left(p_{1}^{12}, p_{3}^{12}\right)=\sqrt{\sum_{i=0}^{g}\left(\alpha_{1 i}^{12}-\alpha_{3 i}^{12}\right)^{2}}=\sqrt{2}
$$

With the Euclidean distance, both preference values $p_{3}^{12}$ and $p_{1}^{12}$ are at the same distance from preference $p_{2}^{12}$, although, it is clear, however, that the first one is further from $p_{2}^{12}$ than the second one. The problem in this case is the way the information of these fuzzy sets is interpreted, as 
a vector of membership values without having taking into account their positions in it. To take into account both the values and positions, a different dissimilarity function able to represent the distribution of the information in the fuzzy set $p_{i}^{l k}$ is necessary. The use of the central value of the fuzzy set, $c v_{i}^{l k}$, is suggested:

$$
c v_{i}^{l k}=\frac{\sum_{h=0}^{g}\left(\operatorname{index}\left(s_{h}^{i}\right)+1\right) \cdot \alpha_{i h}^{l k}}{\sum_{h=0}^{g} \alpha_{i h}^{l k}}, \operatorname{index}\left(s_{h}^{i}\right)=h
$$

This value represents the average position or centre of gravity of the information contained in the fuzzy set $p_{i}^{l k}=\left(\alpha_{i 0}^{l k}, \ldots, \alpha_{i g}^{l k}\right)$. The range of the central value function is the closed interval $[1, g+1]$.

Example 2 The application of (1) to the assessments of example 1 gives the following central values:

$$
c v_{1}^{12}=1, \quad c v_{2}^{12}=4, \quad c v_{3}^{12}=6 .
$$

For $p_{1}^{14}=(0.3,0.8,0.6,0,0,0), p_{1}^{24}=(0,0.3,0.8,0.6,0,0)$, and $p_{1}^{34}=$ $(0,0,0,0.3,0.8,0.6)$, the cental values are:

$$
c v_{1}^{14}=2.18, c v_{1}^{24}=3.18, \text { and } c v_{1}^{34}=5.18 .
$$

As expected, when the information (membership values) moves from the left part of the fuzzy set to the right part, the central value increases.

The value $\left|c v_{i}^{l k}-c v_{j}^{l k}\right|$ can be used as a measure of distance between the the preference values $p_{i}^{l k}$ and $p_{j}^{l k}$, and, therefore, a measure of similarity or proximity between these two preference values, measured in the unit interval $[0,1]$, is defined as:

$$
s\left(p_{i}^{l k}, p_{j}^{l k}\right)=1-\left|\frac{c v_{i}^{l k}-c v_{j}^{l k}}{g}\right|
$$

Clearly, the closer $s\left(p_{i}^{l k}, p_{j}^{l k}\right)$ to 1 the more similar $p_{i}^{l k}$ and $p_{j}^{l k}$ are, while the closer $s\left(p_{i}^{l k}, p_{j}^{l k}\right)$ to 0 the more distant $p_{i}^{l k}$ and $p_{j}^{l k}$ are.

Example 3 The values of similarity between the assessments of example 1 are: $s\left(p_{1}^{12}, p_{2}^{12}\right)=0.4, s\left(p_{1}^{12}, p_{3}^{12}\right)=0$ and $s\left(p_{2}^{12}, p_{3}^{12}\right)=0.6$.

Using the above similarity function (2), the computation of the consensus degrees is carried out in several steps: 
1. After the experts' preferences are uniformed, the central values are calculated:

$$
c v_{i}^{l k} ; \forall i=1, \ldots, m ; \quad l, k=1, \ldots, n \wedge l \neq k
$$

2. For each pair of experts $e_{i}, e_{j}(i<j)$, a similarity matrix $S M_{i j}=$ $\left(s m_{i j}^{l k}\right)$ is calculated, where

$$
s m_{i j}^{l k}=s\left(p_{i}^{l k}, p_{j}^{l k}\right)
$$

3. A consensus matrix, $C M$, is obtained by aggregating all the similarity matrices. This aggregation is carried out at the level of pairs of alternatives:

$$
c m^{l k}=\phi\left(s m_{i j}^{l k}\right) ; i, j=1, \ldots, m \wedge \forall l, k=1, \ldots, n \wedge i<j
$$

In our case, we propose the use of the arithmetic mean as the aggregation function $\phi$, although, different aggregation operators could be used according to the particular properties we want to implement [8].

4. Computation of consensus degrees. As we said in Section 1, the consensus degrees are computed at the three different levels: pairs of alternatives, alternatives and experts.

Level 1. Consensus on pairs of alternatives, $c p^{l k}$, to measure the consensus degree amongst all the experts on each pair of alternatives. In our case, this is expressed by the element $(l, k)$ of the consensus matrix $C M$, i.e.,

$$
c p^{l k}=c m^{l k}, \quad \forall l, k=1, \ldots, n \wedge l \neq k
$$

The closer $c p^{l k}$ to 1 , the greater the agreement amongst all the experts on the pair of alternatives $x_{l}, x_{k}$. This measure will allow the identification of those pairs of alternatives with a poor level of consensus.

Level 2. Consensus on alternatives, $c a^{l}$, to measure the consensus degree amongst all the experts on each alternative. For this, we take the average of each row of the consensus matrix $C M$.

$$
c a^{l}=\frac{\sum_{k=1}^{n} c m^{l k}}{n}
$$

These values can be used to propose modification of preferences associated to those alternatives with a consensus degree lower than a minimal consensus threshold $\gamma$, i.e, $c a^{l}<\gamma$. 
Level 3. Consensus amongst the experts, ce, to measure the global consensus degree amongst the experts' opinions. It is computed as the average of all consensus on alternative values, i.e,

$$
c e=\frac{\sum_{l=1}^{n} c a^{l}}{n}
$$

If the consensus value $c e$ is low then there exists a great discrepancy between the experts' opinions, and therefore they are far from reaching consensus. In this case, the moderator would urge the experts to discuss their opinions further in an effort to bring them closer. However, when the consensus value is high enough, the moderator would finish the consensus process and the selection process would be applied to obtain the final consensus solution to the MPDM problem $[2,15]$.

\subsection{Computation of Proximity Measures}

Proximity measures evaluate the agreement between the individual experts' opinions and the group opinion. Thus, to calculate them, a collective preference relation, $\mathbf{P}_{\mathbf{e}_{\mathbf{c}}}=\left(p_{c}^{l k}\right)$, has to be obtained by means of the aggregation of the set of (uniformed) individual preference relations $\left\{\mathbf{P}_{\mathbf{e}_{\mathbf{i}}}=\left(p_{i}^{l k}\right) ; i=1, \ldots, m\right\}$ :

$$
p_{c}^{l k}=\psi\left(p_{1}^{l k}, \ldots, p_{m}^{l k}\right)
$$

with $\psi$ an "aggregation operator". As $p_{i}^{l k}=\left(\alpha_{i 0}^{l k}, \ldots, \alpha_{i g}^{l k}\right)$ then $p_{c}^{l k}=$ $\left(\alpha_{c 0}^{l k}, \ldots, \alpha_{c g}^{l k}\right)$ with

$$
\alpha_{c j}^{l k}=\psi\left(\alpha_{1 j}^{l k}, \ldots, \alpha_{m j}^{l k}\right) .
$$

which means that $p_{c}^{l k}$ is also a fuzzy set defined on $S_{T}$.

Clearly, the expression (2) can be used to evaluate the agreement between each individual expert's preferences, $\mathbf{P}_{\mathbf{e}_{\mathbf{i}}}$, and the collective preferences, $\mathbf{P}_{\mathbf{e}_{\mathbf{c}}}$. Therefore, the measurement of proximity is carried out in two steps:

1. A proximity matrix, $P M_{i}=\left(p m_{i}^{l k}\right)$, for each expert $e_{i}$, is obtained where $p m_{i}^{l k}=s\left(p_{i}^{l k}, p_{c}^{l k}\right)$.

2. Computation of proximity measures. Again, we calculate proximity measures at three different levels.

Level 1. Proximity on pairs of alternatives, $p p_{i}^{l k}$, to measure the proximity between the preferences, on each pair of alternatives, of each individual expert, $e_{i}$, and the group's ones. In our case, this 
is expressed by the element $(l, k)$ of the proximity matrix $P M_{i}$, i.e.,

$$
p p_{i}^{l k}=p m_{i}^{l k}, \quad \forall l, k=1, \ldots, n \wedge l \neq k
$$

Level 2. Proximity on alternatives, $p a^{l}$, to measure the proximity between the preferences, on each alternative, of each individual expert, $e_{i}$, and the group's ones. For this, we take the average of each row of the proximity matrix $P M_{i}$.

$$
p a_{i}^{l}=\frac{\sum_{k=1}^{n} p p_{i}^{l k}}{n}
$$

Level 3. Experts's proximity, $p e_{i}$, to measure the global proximity between the preferences of each individual expert, $e_{i}$, and the group's ones. It is computed as the average of all proximity on alternative values, i.e,

$$
p e_{i}=\frac{\sum_{l=1}^{n} p a_{i}^{l}}{n}
$$

If the above values are close to 1 then they have a positive contribution for the consensus to be high, while if they are close to 0 then they have a negative contribution to consensus. As a consequence, these proximity measures can be used to build a feedback mechanism, based on simple rules or recommendations to support the experts in changing their opinions and thus obtain the highest degree of consensus possible, as was done in [8].

\section{Conclusions}

The reaching of consensus in GDM problems needs measurements to assess the consensus degree between the experts. In this paper, two types of measurements were proposed: consensus measurement and proximity measurement. The first one is used to assess the agreement amongst all the experts' opinions, while the second one is used to find out how far the individual opinions are from the group opinion. Both types of measurements are computed at three different levels of representation of information: pair of alternatives, alternatives and experts.

We have also shown that to make the measurement of consensus possible in multi-granular GDM problems, it was necessary to unify the different linguistic term sets into a single linguistic term set. To do this, fuzzy sets defined on a basic linguistic term set (BLTS) were used. 
Finally, for future research, the proximity measures will be used to design a consensus support system able to generate advice on the necessary changes in the experts' opinions in order to reach consensus, which would make the figure of the moderator unnecessary in the consensus reaching process.

\section{References}

1. Bonissone, P.P., Decker, K.S.: Selecting Uncertainty Calculi and Granularity: An Experiment in Trading-off Precision and Complexity, in: L.H. Kanal and J.F. Lemmer, Eds., Uncertainty in Artificial Intelligence (North-Holland, 1986) 217-247

2. Bryson, N.: Group decision-making and the analytic hierarchy process: exploring the consensus-relevant information content. Computers and Operational Research 23 (1996) 27-35.

3. Fodor, J., Roubens, M.: Fuzzy Preference Modelling and Multicriteria Decision Support (Kluwer Academic Publishers, Dordrecht, 1994).

4. Herrera, F., Herrera-Viedma, E., Martínez, L.: A fusion aproach for managing multi-granularity linguistic term sets in decision making. Fuzzy Sets and System 114 (2000) 43-58

5. Herrera, F., Herrera-Viedma, E., Verdegay, J.L.: Linguistic Measures Based on Fuzzy Coincidence for Reaching Consensus in Group Decision Making. Int. J. of Approximate Reasoning (1997) 309-334

6. Herrera-Viedma, E.: Modeling the retrieval process for an information retrieval system using an ordinal fuzzy linguistic approach. J. of the American Society for Information Science and Technology 52:6 (2001) 460-475

7. Herrera-Viedma, E., Cordon, O., Luque, M., Lopez, A.G., Muñoz, A.N.: A Model of Fuzzy Linguistic IRS Based on Multi-Granular Linguistic Information. Int. J. of Approximate Reasoning 34 (3) (2003) 221-239

8. Herrera-Viedma, E., Herrera, F., Chiclana, F.: A Consensus Model for Multiperson Decision Making with Different Preference Structures. IEEE Transactions on Systems, Man and Cybernetics-Part A: Systems and Humans 32 (2002) 394-402

9. Kacprzyk, J., Nurmi, H., Fedrizzi, M., Eds: Consensus under Fuzziness. (Boston: Kluwer Academic Publishers, 1997)

10. Marimin, Umano, M., Hatono I., Tamure, H.: Linguistic labels for expressing fuzzy preference relations in fuzzy group decision making. IEEE Transactions on Systems, Man and Cybernetics-Part B: Cybernetics 28 (1998) 205-218

11. Miller, G.A.: The magical number seven or minus two: some limits on our capacity of processing information. Psychological Rev. 63 (1956) 81-97.

12. Torra, V.: Aggregation of linguistic labels when semantics is based on antonyms Int. J. of Intelligent System 16 (2001) 513-524

13. Yager, R.R.: An Approach to Ordinal Decision Making. Int. J. of Approximate Reasoning 12 (1995) 237-261

14. Zadeh, L.A.: The Concept of a Linguistic Variable and Its Applications to Approximate Reasoning. Part I, Information Sciences 8 (1975) 199-249. Part II, Information Sciences 8 (1975) 301-357. Part III, Information Sciences 9 (1975) 43-80

15. Zadrozny, S.: An Approach to the Consensus Reaching Support in Fuzzy Environment. In J. Kacprzyk, H. Nurmi and M. Fedrizzi. Eds., Consensus under Fuzziness, (Kluwer Academic Publishers, Boston, 1997) 83-109 